\title{
El manejo ambiental en las plantas de tratamiento de aguas residuales en el Perú
}

\author{
Mg. Alberto Cieza Perez \\ cipereza@ucvvirtual.edu.pe \\ https://orcid.org/0000-0002-4144-0948 \\ Dr. Marcelino Callao Alarcón \\ calarconm@ucvvirtual.edu.pe \\ https://orcid.org/000-0001-7295-2375 \\ Msc. Mirtha Culqui Lozada \\ clozadam@ucvvirtual.edu.pe \\ https://orcid.org/0000-0002-8780-9755
}

Mg. Jorge Antonio Malca Florindes mflorindesj@ucvvirtual.edu.pe https://orcid.org/0000-0002-1090-6389

Mg. Víctor Hugo Puican Rodriguez victor-puican@ hotmail.com https://orcid.org/0000-0001-7402-9576

Universidad César Vallejo

Chiclayo - Perú

\section{RESUMEN}

Se llevó a cabo una revisión documental sobre la producción y publicación de trabajos de investigación referentes al estudio de la variable El manejo ambiental en las plantas de tratamiento de aguas residuales. El propósito del análisis bibliométrico propuesto en el presente documento, es conocer las características principales del volumen de publicaciones registradas en base de datos Scopus durante el periodo 2015-2020 en países Latinoamericanos, logrando la identificación de 908 publicaciones en total. La información suministrada por dicha plataforma, fue organizada mediante gráficos y figuras categorizando la información por el Año de Publicación, País de Origen, Área de Conocimiento y Tipo de Publicación. Una vez descritas dichas características, se hace referencia mediante un análisis cualitativo, la postura de diferentes autores frente a la temática propuesta. Dentro de los principales hallazgos realizados por medio de la 
presente investigación, se encuentra que Brasil con 626 publicaciones, es el país latinoamericano con mayor producción. El Área de Conocimiento que mayor aporte hizo a la construcción de material bibliográfico referente al estudio de El manejo ambiental en las plantas de tratamiento de aguas residuales fue ciencias ambientales con 623 documentos publicados, y el Tipo de Publicación que más fue usado durante el periodo señalado anteriormente fue el Artículo de Revista que representan el 89\% de la producción científica total.

Palabras claves: manejo ambiental; tratamiento de aguas residuales. 


\title{
Environmental management at wastewater treatment plants in Peru
}

\begin{abstract}
A documentary review was carried out on the production and publication of research papers related to the study of the variable environmental management in wastewater treatment plants. The purpose of the bibliometric analysis proposed in this document, is to know the main characteristics of the volume of publications registered in Scopus database during the period 2015-2020 in Latin American countries, achieving the identification of 908 publications in total. The information provided by said platform, was organized by means of graphs and figures categorizing the information by Year of Publication, Country of Origin, Area of Knowledge and Type of Publication. Once these characteristics were described, a qualitative analysis was used to refer to the position of different authors on the proposed topic. Among the main findings of this research, it is found that Brazil, with 626 publications, is the Latin American country with the highest production. The area of knowledge that made the greatest contribution to the construction of bibliographic material referring to the study of environmental management in wastewater treatment plants was environmental sciences with 623 published documents, and the type of publication that was most used during the period mentioned above was the journal article, which represents $89 \%$ of the total scientific production.
\end{abstract}

Key words: environmental management; wastewater treatment.

Artículo recibido: 02 noviembre. 2021 Aceptado para publicación: 28 noviembre 2021 Correspondencia: cipereza@ucvvirtual.edu.pe Conflictos de Interés: Ninguna que declarar 


\section{INTRODUCCIÓN}

La gestión ambiental en las plantas de tratamiento de aguas residuales representa un gran número de ventajas en la optimización de los procesos que se realizan en estas, al usar los recursos de manera más eficiente, aprovechando de la mejor forma y reduciendo la emisión y contaminación ayudando así a implementar políticas que hagan a la gestión de estas empresas más sostenibles, lo cual se traduce en innovación empresarial haciendo a estas compañías más competentes alineadas a los objetivos del desarrollo sostenible de los que habla la ONU. Un ejemplo de las propuestas de gestión ambiental en el tratamiento de aguas residuales se encuentra en el trabajo titulado "Propuesta Técnica de Gestión Ambiental Sostenible para el Aprovechamiento de Lodos que Provienen de las Plantas de Tratamiento de Aguas Residuales Domésticas en Campamentos Mineros del Perú" (Onofre Aquino, 2019) en esta investigación se trata la problemática de los lodos en los campamentos mineros del Perú, ya que estos no son tratados de una manera correcta para su aprovechamiento teniendo en cuenta que las empresas mineras toman ventajas de ciertos vacíos legales para no implementar una planta de tratamiento de aguas residuales integrales. Por lo que en este estudio propone alternativas de aprovechamiento del lodo como elemento primario para obtener abonos y fertilizantes como una estrategia para hacer más sostenible estos procesos.

en el tratamiento de las aguas residuales, se tienen varios riesgos de contaminación ambiental al verter estos desechos a las aguas del mar, lo que genera una modificación en el medio ambiente y el cambio del suelo además de afectaciones a la salud de las comunidades que residen cerca de los puntos de vertido de estos desechos; por estas razones Cerna (2012) considera que la empresas prestadoras de servicio de agua potable y alcantarillado, deben iniciar la inclusión en sus empresas de una política ambiental, que conlleve a la elaboración de planes de Gestión Ambiental; con el objeto de proteger la salud, la seguridad y la calidad de vida de los usuarios y población en general. Esto hablando específicamente de las 50 empresas prestadoras de este servicio en Perú. Por lo anterior, es importante conocer en materia de recursos bibliográficos, el estado actual de la investigación referente a El manejo ambiental en las plantas de tratamiento de aguas residuales, por lo que se propone un análisis bibliométrico de la producción científica registrada en base de datos Scopus durante el periodo 2015-2020 que permita dar respuesta a la pregunta ¿ Cómo ha sido la producción y publicación de 
trabajos de investigación relacionados al estudio de la variable manejo ambiental en las plantas de tratamiento de aguas residuales durante el periodo 2015-2020?

\section{ESTRATEGIAS METODOLÓGICAS O MATERIALES Y MÉTODOS}

\subsection{Objetivo general}

Analizar desde una perspectiva bibliométrica y bibliográfica, la producción de trabajos de investigación de alto impacto sobre la variable el manejo ambiental en las plantas de tratamiento de aguas residuales durante el periodo 2015-2020.

\subsection{Metodología}

Se realiza análisis cuantitativo de la información suministrada por Scopus bajo un enfoque bibliométrico sobre la producción científica referente a El manejo ambiental en las plantas de tratamiento de aguas residuales. Asimismo, se analiza desde una perspectiva cualitativa, ejemplos sobre algunos trabajos de investigación publicados en el área de estudio señalada anteriormente, desde un enfoque bibliográfico para describir la postura de diferentes autores frente al tema propuesto.

La búsqueda se realiza a través de la herramienta suministrada por Scopus y se establecen parámetros referenciados en la Tabla 1.

\subsection{Diseño metodológico}

\begin{tabular}{|c|c|c|c|}
\hline & FASE & DESCRIPCIÓN & CLASIFICACIÓN \\
\hline FASE 1 & $\begin{array}{l}\text { Levantamiento de } \\
\text { datos }\end{array}$ & $\begin{array}{l}\text { Se realiza el } \\
\text { levantamiento de } \\
\text { datos por medio de la } \\
\text { herramienta } \\
\text { Búsqueda en la } \\
\text { página web de } \\
\text { Scopus, por medio de } \\
\text { la cual se logra la } \\
\text { identificación de un } \\
\text { total de } \\
\text { publicaciones. }\end{array}$ & $\begin{array}{l}\text { Documentos } \\
\text { publicados cuyas } \\
\text { variables de estudio } \\
\text { están relacionadas a El } \\
\text { manejo ambiental en } \\
\text { las plantas de } \\
\text { tratamiento de aguas } \\
\text { residuales. } \\
\text { Trabajos } \\
\text { investigación } \\
\text { publicados durante el } \\
\text { Periodo 2015-2020. } \\
\text { Limitado a países } \\
\text { latinoamericanos. } \\
\text { Sin distinción de área } \\
\text { de conocimiento. } \\
\text { Sin distinción de tipo } \\
\text { de publicación. }\end{array}$ \\
\hline
\end{tabular}




\begin{tabular}{|c|c|c|c|}
\hline FASE 2 & $\begin{array}{l}\text { Contrucción de } \\
\text { material de análisis }\end{array}$ & $\begin{array}{l}\text { Se procede a la } \\
\text { organización de la } \\
\text { información } \\
\text { identificada en la fase } \\
\text { anterior. } \\
\text { clasificación se hará } \\
\text { mediante gráficos, } \\
\text { figuras y tablas a } \\
\text { partir de datos } \\
\text { suministrados por } \\
\text { Scopus. }\end{array}$ & $\begin{array}{l}\text { Co-ocurrencia de } \\
\text { Palabras. } \\
\text { Año de publicación } \\
\text { País de origen de la } \\
\text { publicación. } \\
\text { Área de } \\
\text { conocimiento. } \\
\text { Tipo de Publicación }\end{array}$ \\
\hline FASE 3 & $\begin{array}{lr}\text { Redacción de las } \\
\text { conclusiones } \\
\text { documento final }\end{array}$ & $\begin{array}{l}\text { Luego del análisis } \\
\text { realizado en la fase } \\
\text { anterior, se procede a } \\
\text { la redacción de las } \\
\text { conclusiones } \\
\text { elaboración } \\
\text { documento final. }\end{array}$ & \\
\hline
\end{tabular}

Tabla 1. Diseño metodológico.

Fuente: Elaboración propia (2021)

\section{RESULTADOS}

\subsection{Co-ocurrencia de palabras}

La Figura 1 muestra la co-ocurrencia de palabras clave dentro de las publicaciones identificadas en base de datos Scopus.

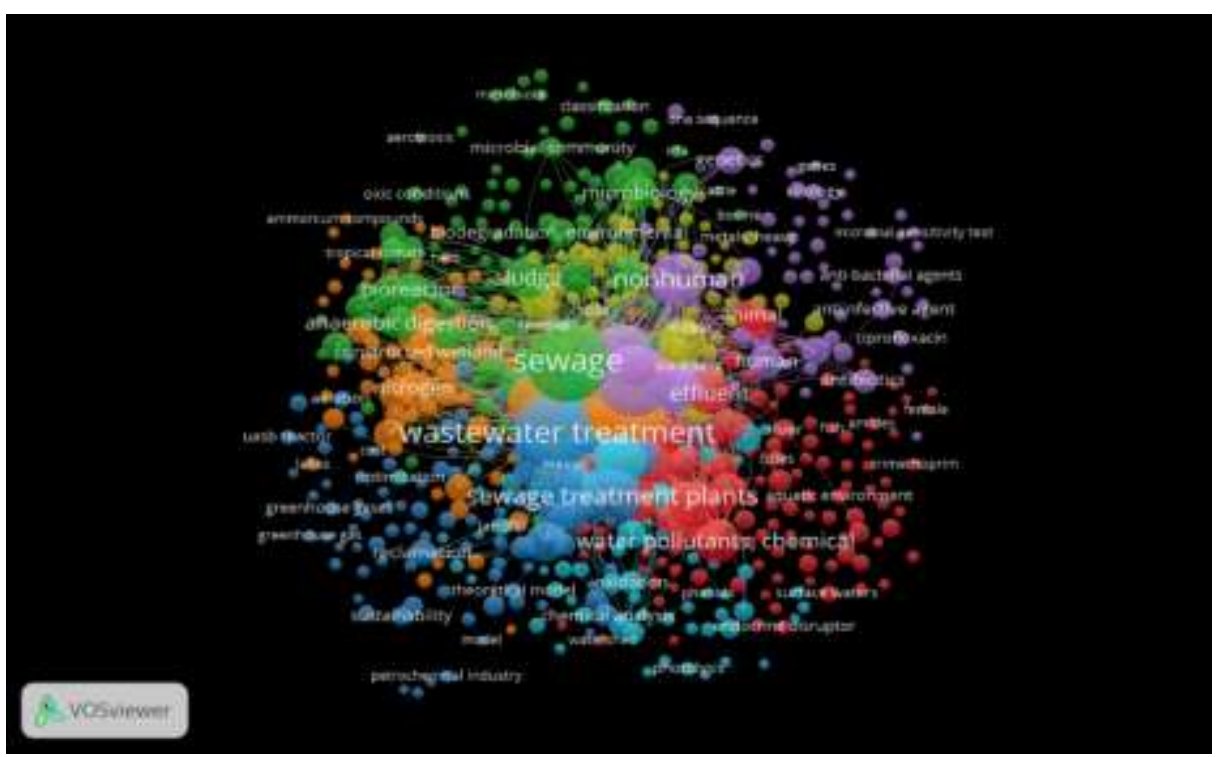

Figura 1. Co-ocurrencia de palabras

Fuente: Elaboración propia (2021); a partir de datos suministrados por Scopus. 
Como lo muestra la figura 1, la palabra clave más utilizada es aguas residuales el cual es la variable central de este estudio al estudiar el tratamiento en las plantas de estas aguas y determinar el impacto de la gestión ambiental en el cambio de procedimientos con el fin de ser más amigables con el medio ambiente. Con esta palabra clave se encuentran relacionada contaminación química del agua, gases invernadero e industria petroquímica las cuales hacen referencia a los principales daños que causa el mal manejo de las aguas residuales al aumentar la contaminación en los ríos y mares gracias al mal tratamiento de los desechos industriales y petroquímicos que se generan en la explotación minera. Por parte de gestión ambiental encontramos palabras claves como sostenibilidad, modelo teórico, agentes anti-bacterianos, optimización y costos los cuales hacen alusión a los objetivos que persigue la implementación de planes ambientales a las plantas de tratamiento de aguas residuales al reducir su impacto medioambiental, optimizar procesos y a su vez reducir costos que permitan el hacer estos procesos más sostenibles que le saquen el mayor provecho a estos residuos.

\subsection{Distribución de la producción científica por año de publicación.}

La Figura 2 muestra cómo está distribuida la producción científica según el año de publicación, teniendo en cuenta que se toma el periodo comprendido entre los años 2015 y 2020.

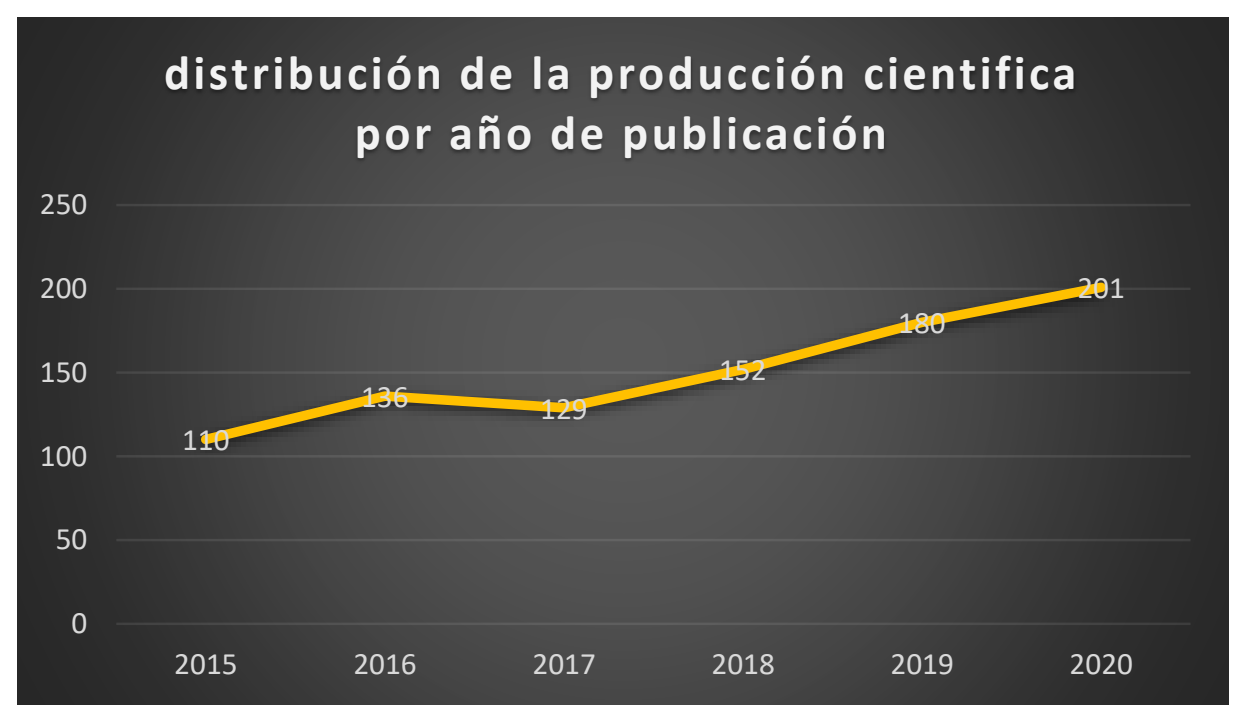

Figura 2. Distribución de la producción científica por año de publicación.

Fuente: Elaboración propia (2021); a partir de datos suministrados por Scopus.

El año 2020, fue el año co mayor número de documentos publicados registrados, dentro de los cuales se encuentra "Análisis de la eficiencia de las plantas de tratamiento de 
aguas residuales: el problema de la definición de resultados deseables y su solución" (Fuentes, Molinos-Senante, Hernández-Sancho, \& Sala-Garrido, 2020) en este estudio se evalúan las plantas de tratamiento de aguas residuales para determinar su desempeño económico y ambiental. Por lo que se evidenció que los puntajes de eficiencia se ven afectados por la selección de salida. Por lo que esta investigación sugiere la modificación del diseño y mantenimiento de las plantas con el fin de hacerlas más sustentables y a su vez encontrar la forma de reducir costos en sus procesos siendo estos cada vez más eficaces utilizando una menor cantidad de presupuesto.

En segundo lugar, se encuentra 2019 con un total de 180 publicaciones relacionadas a las variables en estudio, dentro de estos podemos identificar "Tratamiento de aguas negras mediante un reactor de manto de lodo anaeróbico de flujo ascendente (UASB): un estudio piloto" (Mohedano, Tonon, Costa, Pelissari, \& Belli, 2019) en esta investigación se presenta un plan piloto que busca determinar la emisión de gases de efecto invernadero de la plantas de tratamiento de aguas residuales como una alternativa sostenible, en donde se utilizan estanques de lenteja de agua. Por lo que se evidenció que con la utilización de este sistema no se emitía gas metano y se reducía 3 veces la emisión de dióxido de carbono (CO2) por lo que la hace una alternativa más sostenible que implementa la tecnología en sus procesos.

\subsection{Distribución de la producción científica por país de origen.}

La Figura 3 muestra cómo está distribuida la producción científica según la nacionalidad de los autores.

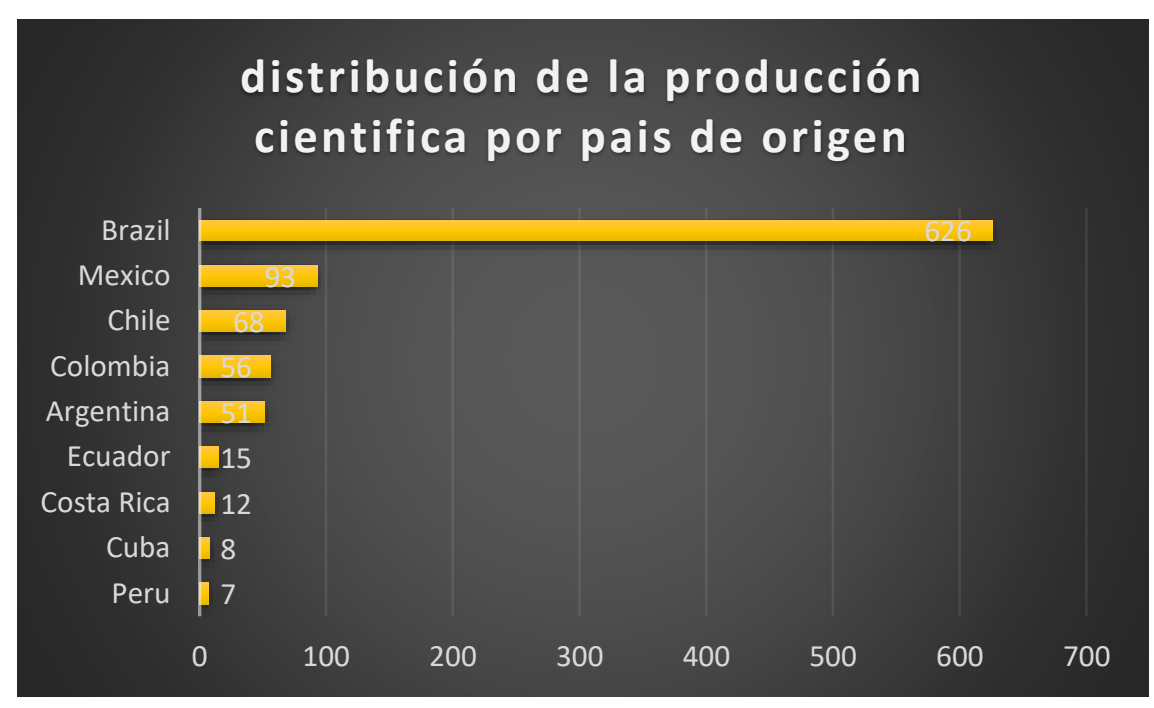

Figura 3. Distribución de la producción científica por país de origen.

Fuente: Elaboración propia (2021); a partir de datos suministrados por Scopus. 
Como lo muestra la figura 3, Brasil es el país latinoamericano con mayor aporte en investigaciones relacionadas al Manejo Ambiental en las a Plantas de tratamiento de Aguas residuales con un total de 626 documentos registrados en Scopus, dentro de estas publicaciones se encuentra "Seguimiento de la ocurrencia de fármacos psicotrópicos en plantas de tratamiento de aguas residuales y aguas superficiales brasileñas, con evaluación de riesgos ambientales" (Pivetta, Rodrigues-Silva, Ribeiro, \& Rath, 2020) esta investigación analiza cómo llegan a las plantas de tratamiento de aguas residuales algunos psicotrópicos, donde pueden ser eliminadas de forma incompleta durante el tratamiento, lo que lleva a la contaminación de las aguas superficiales, por lo que realizaron un estudio de la presencia de 10 fármacos de esta clase después de ser tratada las aguas residuales, en donde se concluyó que si hay presencia de estos fármacos pero que no representan un problema para la conservación de la biota acuática, aun así se presenta la necesidad de implementar nuevos planes ambientales que ayuden a mitigar los efectos que esto podría representar a largo plazo.

En este punto cabe resaltar que la producción de publicaciones científicas al ser clasificada por país de origen, presenta una característica especial y es la colaboración entre autores con diferente afiliación a instituciones tanto públicas como privadas, y estas instituciones pueden ser del mismo país o de diferentes nacionalidades por lo que la producción de un artículo con coautoría de diferentes autores de distintos países de origen permite a cada uno de los países sumar como una unidad en la publicaciones generales. Lo anterior se explica de una mejor manera en la Figura 4 donde se observa el flujo de trabajos en colaboración de diferentes países.

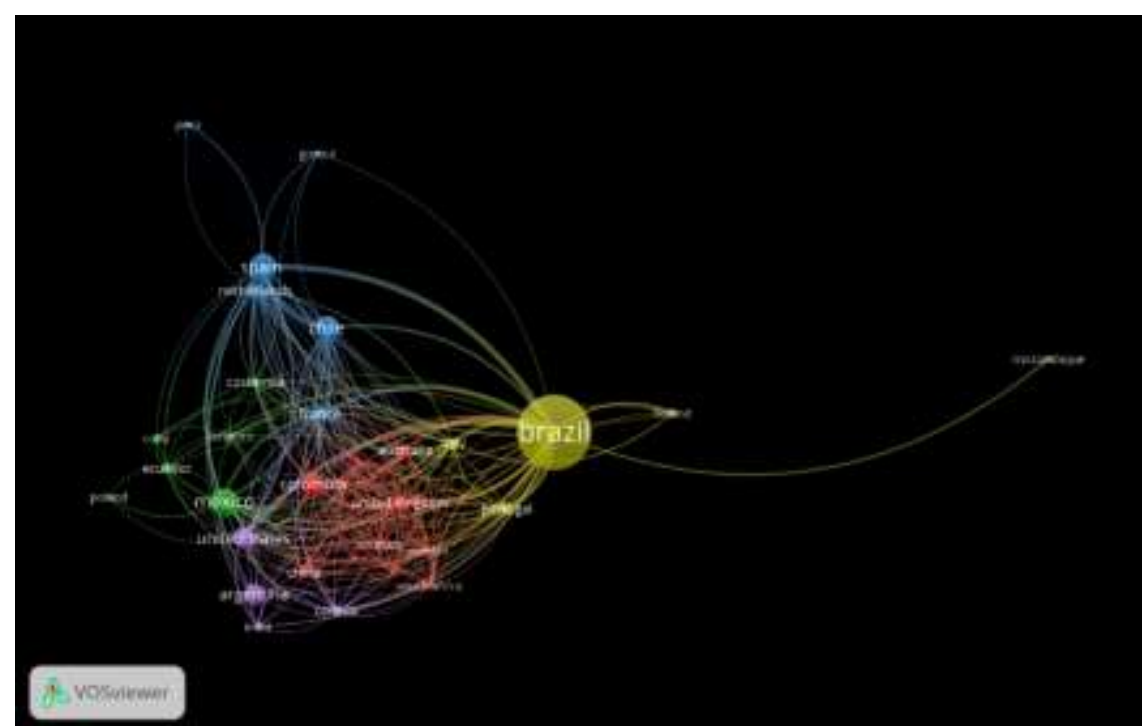

Figura 4. Co-citaciones entre países.

Fuente: Elaboración propia (2021); a partir de datos suministrados por Scopus 
Como se mencionó anteriormente, Brasil es el país latinoamericano con mayor número de publicaciones relacionadas a las variables en estudio presentando publicaciones con autores afiliados a organizaciones pertenecientes a países que no son latinoamericanos, como por ejemplo, Portugal, Finlandia, Italia y Mozambique. En segundo lugar se encuentra México con 93 publicaciones en coautoría con afiliados a organizaciones de Estados unidos, España, Francia y Reino unido; dentro de estos documentos se encuentra el artículo titulado "Aguas residuales de megaciudades vertidas en una cuenca cercana: buscando escenarios sostenibles en un estudio de caso" (Chamizo-Checa, y otros, 2020) donde se determina que El alcantarillado de las megaciudades genera dependencia socioeconómica relacionada con la disponibilidad de agua en áreas cercanas, especialmente en países con estrés hídrico, por lo que Se utilizó el Sistema de Evaluación y Planificación del Agua (WEAP) para estimar la demanda y el suministro de agua; dando como resultado que las políticas actuales comprometen las demandas futuras del Valle por lo que se evidencia la necesidad de implementar nuevas estrategias ambientales que permitan contrarrestar estos efectos negativos a los recursos de las generaciones futuras.

\subsection{Distribución de la producción científica por área de conocimiento}

A continuación, se muestra en la Figura 5, cómo está distribuida la producción de publicaciones científicas según el área de conocimiento por medio de la cual se ejecutan las diferentes metodologías de investigación.

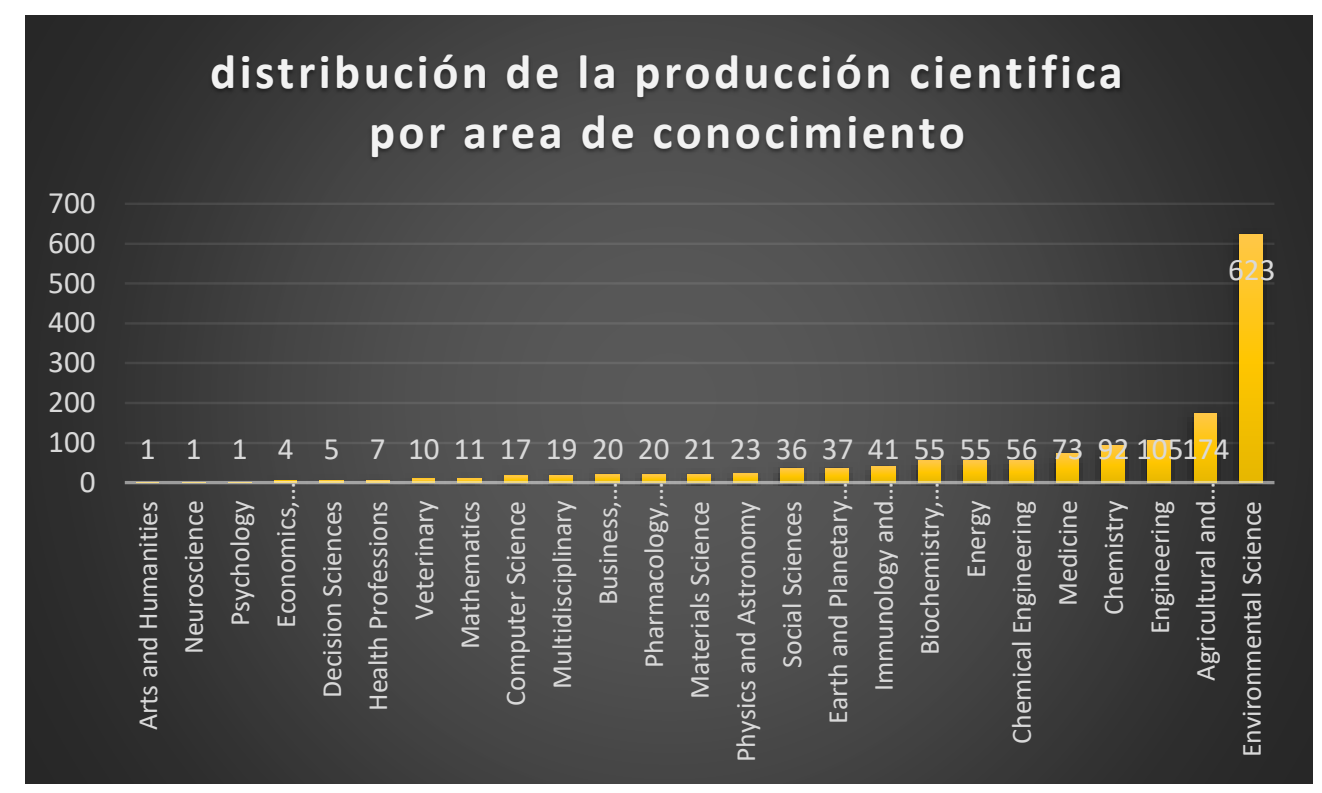

Figura 5. Distribución de la producción científica por área de conocimiento.

Fuente: Elaboración propia (2021); a partir de datos suministrados por Scopus. 
El área de conocimiento que mayor aporte hizo al estudio de la gestión ambiental de las plantas de tratamiento de aguas residuales fue ciencias ambientales debido a la naturaleza de la investigación con un total de 623 documentos, dentro de los cuales se encuentra "Evaluación y optimización del uso de un nuevo coagulante natural (Guazuma ulmifolia) para el tratamiento de aguas residuales lácteas" (Muniz, Silva, \& Borges, 2020) el usos de Guazuma ulmifolia se presenta como una alternativa para el tratamiento de las aguas residuales lácteas, por lo que este estudio dio como resultado la evidencia de la novedad, eficiencia, bajo costo y propiedades ecológicas como alternativa para el tratamiento de aguas residuales lácteas en la optimización del tratamiento de estas aguas residuales utilizando un presupuesto menor garantizando la sostenibilidad del servicio. En segundo lugar se encuentra ciencias agriculturales y biológicas con 124 documentos en total, dentro de estos podemos identificar "Reutilización del agua y horticultura urbana: Alianza hacia ciudades más sostenibles" (Bizari \& Cardoso, 2020) esta investigación plantea la necesidad de repensar los modelos actuales de uso de los recursos hídricos, por lo que el uso de agua reciclada procedente de aguas residuales domésticas tratadas en actividades agrícolas está ganando terreno, ya que al implementar esta medida se busca la protección ambiental y una alta productividad agrícola; La creciente urbanización contribuye a generar, pero también a fomentar el tratamiento y uso de aguas residuales, ya que las áreas urbanas son, en un tiempo, principales consumidores de agua y principales productores de aguas residuales, por lo que la re utilización de estas aguas se presenta como un plan ambiental que busca mitigar los desechos generados en las zonas urbanas aprovechando en los procesos de agricultura, haciendo así estos procesos renovables y hasta en su momento podrían ser autosustentables. 


\subsection{Tipo de publicación}

La Figura 6 muestra cómo está distribuida la producción bibliográfica según el tipo de publicación escogida por los autores.

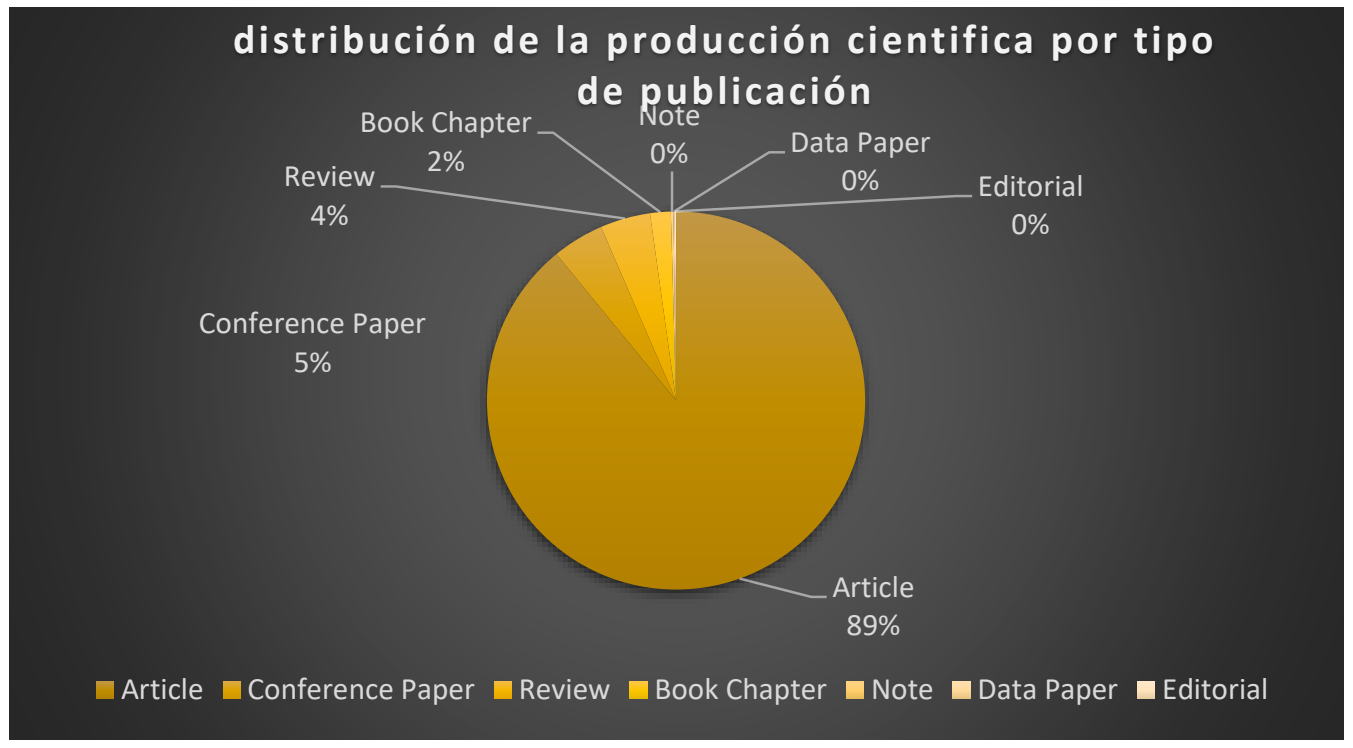

Figura 6. Tipo de publicación

Fuente: Elaboración propia (2021); a partir de datos suministrados por Scopus.

Como lo muestra la Figura 6, dentro de los diferentes tipos de publicaciones, el 89\% del total de documentos identificados mediante la Fase 1 del Diseño Metodológico, corresponden a Artículos de Revista. En segundo lugar se encuentran las actas de conferencia las cuales representan el 5\% del total de las publicaciones registradas dentro de las cuales se encuentra "Efectos de las aguas residuales en los ambientes naturales de la región amazónica (Pará-Brasil)" (Sousa, Monteiro, Gorayeb, Costa, \& Pereira, 2016) donde se analizaron los efectos negativos de la descarga de aguas residuales en dos ambientes amazónicos, el estuario del Caeté y el río Cereja en donde se encontraron altos niveles de nitrógeno y coliformes fecales. Por lo que se concluye que la construcción de un sistema público de alcantarillado y una planta de tratamiento por parte de las autoridades locales será un paso clave hacia la mejora de la calidad del agua de los ríos y ayudaría a mitigar la contaminación hídrica. En tercer lugar se encuentran las revisiones representando el $4 \%$ de los documentos registrados en Scopus, dentro de estas publicaciones se encuentra la titulada "Epidemiología basada en aguas residuales (Wbe) y detección viral en aguas superficiales contaminadas: una herramienta valiosa para la vigilancia del covid-19: una breve revisión" (Aguiar-Oliveira, y otros, 2020) en 
esta investigación se plantea la transmisión fecal-oral del SARS-CoV-2 ya que se elimina en las excretas humanas y luego se elimina en el alcantarillado o en el medio ambiente, en entornos de saneamiento básico deficientes por lo que en entornos de saneamiento deficiente, los ríos contaminados podrían utilizarse alternativamente como fuente de vigilancia ambiental.

\section{CONCLUSIONES O CONSIDERACIONES FINALES}

Gracias al análisis bibliométrico propuesto en la presente investigación, se puede determinar que Brasil es el país de Latinoamérica con mayor número de registros bibliográficos en base de datos Scopus durante el periodo comprendido entre los años 2015 y 2020 con un total de 626 documentos. La producción científica relacionada al estudio de El Manejo Ambiental en las Plantas de Tratamiento de Aguas Residuales, ha presentado un crecimiento importante durante el periodo anteriormente señalado, pasando de 110 publicaciones en 2015 a 201 unidades en 2020, es decir se logró duplicar la creación de registros bibliográficos en un periodo de 5 años, lo que indica la importancia la gestión ambiental representa en las nuevas medidas tomadas por las plantas de tratamiento de aguas residuales con el fin de mitigar los efectos negativos que representan estas aguas al medio ambiente y a la calidad del servicio.

El manejo ambiental en las plantas de tratamiento de aguas residuales es de gran importancia ya que busca integrar nuevas medidas y optimizar sus procesos a través de herramientas que ayuden a convertirlo en un procedimiento más amigable con el medio ambiente siendo así más sostenible y brindándole a la comunidad una mejor calidad en los recursos hídricos y en la salud pública. Además de esto la gestión ambiental es pieza clave en la reducción en costos de operación al implementar procesos más eficientes con menor presupuesto ya que la mayoría de estos planes ambientales parten de la reutilización de las aguas residuales con el fin de obtener el mejor provecho de estos desechos. Por lo anterior se logra concluir la importancia de la implementación de la gestión ambiental en las compañías encargadas del tratamiento de las aguas residuales al ser esta la herramienta que ayuda a implementar nuevas políticas que vayan encaminadas a lograr una sostenibilidad empresarial la cual genere un equilibrio entre el crecimiento económico y la protección de los recursos naturales que resultan afectados en estos procedimientos, además de generar nuevas formas de aprovechamiento de estos residuos. Es por eso que se resalta la necesidad de estudios como el presentado en el 
presente documento, que realicen un recorrido por aquellos textos que aborden el mencionado tema, con el fin de dar al lector una visión amplia de la situación actual de la bibliografía sobre El manejo ambiental en las plantas de tratamiento de aguas residuales.

\section{LISTA DE REFERENCIAS}

Aguiar-Oliveira, M., Campos, A., Matos, A., Rigotto, C., Sotero-Martins, A., Teixeira, P., \& Siqueira, M. (2020). Wastewater-based epidemiology (Wbe) and viral detection in polluted surface water: A valuable tool for covid-19 surveillance-a brief review. International Journal of Environmental Research and Public Health , 1 - 19 .

Bizari, D., \& Cardoso, J. (2020). Reuse water and urban horticulture: Alliance towards more sustainable cities. Horticultura Brasileira , 311 - 317.

Cerna Rubio, F. E. (2012). Contaminación de la bahía "el ferrol" con aguas residuales domésticas y propuesta de gestión ambiental. Universidad nacional de trujillo.

Chamizo-Checa, S., Otazo-Sánchez, E., Gordillo-Martínez, A., J., S.-S., GonzálezRamírez, C., \& Muñoz-Nava, H. (2020). Megacity wastewater poured into a nearby basin: Looking for sustainable scenarios in a case study. Water (Switzerland).

Fuentes, R., Molinos-Senante, M., Hernández-Sancho, F., \& Sala-Garrido, R. (2020). Analysing the efficiency of wastewater treatment plants: The problem of the definition of desirable outputs and its solution. Journal of Cleaner Production.

Mohedano, R., Tonon, G., Costa, R., Pelissari, C., \& Belli, F. P. (2019). Black water treatment by an upflow anaerobic sludge blanket (UASB) reactor: A pilot study. Science of the Total Environment, 1043 - 1050.

Muniz, G., Silva, T., \& Borges, A. (2020). Assessment and optimization of the use of a novel natural coagulant (Guazuma ulmifolia) for dairy wastewater treatment. Science of the Total Environment.

Onofre Aquino, E. F. (2019). Propuesta Técnica de Gestión Ambiental Sostenible para el Aprovechamiento de Lodos que Provienen de las Plantas de Tratamiento de Aguas Residuales Domésticas en Campamentos Mineros del Perú. Pontificia Universidad Catolica del Peru - CENTRUM . 
Pivetta, R., Rodrigues-Silva, C., Ribeiro, A., \& Rath, S. (2020). Tracking the occurrence of psychotropic pharmaceuticals in Brazilian wastewater treatment plants and surface water, with assessment of environmental risks. Science of the Total Environment.

Sousa, N., Monteiro, M., Gorayeb, Costa, R., \& Pereira, L. (2016). Effects of sewage on natural environments of the amazon region (Pará-Brazil). 4th International Coastal Symposium, ICS 2016 (págs. 158-162). Journal of Coastal Research.

Abd-Elmaksoud, S., Castro-del Campo, N., Gerba, C. P., Pepper, I. L., \& Bright, K. R. (2019). Comparative assessment of BGM and PLC/PRF/5 cell lines for enteric virus detection in biosolids. Food and Environmental Virology, 11(1), 32-39. doi:10.1007/s12560-019-09366-4

Achon, C. L., Coimbra, P. R. S., \& Kellner, E. (2019). Influence of the geotextile blanket and sludge height in anaerobic sludge dewatering using drainage bed. [Influência da manta geotêxtil e altura da camada de lodo no desaguamento de lodo anaeróbio em leito de drenagem] Engenharia Sanitaria e Ambiental, 24(4), 849-860. doi:10.1590/s1413-41522019184786

Adewuyi, A., \& Pereira, F. V. (2018). Preparation and application of EDTAfunctionalized underutilized adansonia digitata seed for removal of cu(II) from aqueous solution. Sustainable Environment Research, 28(3), 111-120. doi:10.1016/j.serj.2017.12.002

Agnelo, L., Leonel, L. P., Silva, N. B., Candello, F. P., Schneider, J., \& Tonetti, A. L. (2020). Effects of wastewater disinfectants on the soil: Implications for soil microbial and chemical attributes. Science of the Total Environment, 706 doi:10.1016/j.scitotenv.2019.136007

Aguilar Aguilar, F. A., Nelson, D. L., Pantoja, L. D. A., \& Soares Dos Santos, A. (2017). Study of anaerobic co-digestion of crude glycerol and swine manure for the production of biogas. Revista Virtual De Química, 9(6), 2384-2403. doi:10.21577/1984-6835.20170142

Aguilar-Benitez, I., \& Blanco, P. A. (2018). Methane recovery and reduction of greenhouse gas emissions: WWTP nuevo laredo, tamaulipas, mexico. [Recuperación de metano y reducción de emisiones en PTAR Nuevo Laredo, 
Tamaulipas, México] Tecnología y Ciencias Del Agua, 9(2), 86-111. doi:10.24850/j-tyca-2018-02-04

Busto, R. V., Roberts, J., Hunter, C., Escudero, A., Helwig, K., \& Coelho, L. H. G. (2020). Mechanistic and ecotoxicological studies of amoxicillin removal through anaerobic degradation systems. Ecotoxicology and Environmental Safety, 192 doi:10.1016/j.ecoenv.2020.110207

Bustos, Y. A., Rangel-Peraza, J. G., Rojas-Valencia, M. N., Bandala, E. R., ÁlvarezGallegos, A., \& Vargas-Estrada, L. (2016). Treatment of industrial effluents by electrochemical generation of $\mathrm{H} 2 \mathrm{O} 2$ using an $\mathrm{RVC}$ cathode in a parallel plate reactor. Environmental Technology (United Kingdom), 37(7), 815-827. doi:10.1080/09593330.2015.1086820

Butassi, E., Raimondi, M., Postigo, A., Cordisco, E., \& Sortino, M. (2017). Antimicrobial activity testing techniques. Essential oils and nanotechnology for treatment of microbial diseases (pp. 298-309) doi:10.1201/9781315209241 Retrieved from www.scopus.com

Cabrol, L., Urra, J., Rosenkranz, F., Kroff, P. A., Plugge, C. M., Lesty, Y., \& Chamy, R. (2015). Influence of phenylacetic acid pulses on anaerobic digestion performance and archaeal community structure in WWTP sewage sludge digesters. Water Science and Technology, 71(12), 1790-1799. doi:10.2166/wst.2015.165

Caceres, E., \& Alca, J. J. (2016). Potential for energy recovery from A wastewater treatment plant. IEEE Latin America Transactions, 14(7), 3316-3321. doi:10.1109/TLA.2016.7587636

Cáceres, J. R., Rojas, J. P., \& Sánchez, J. (2019). Determination of expandability clay from san josé de cúcuta metropolitan area, by adding different percentages of sewage sludge. Paper presented at the Journal of Physics: Conference Series, , 1388(1) doi:10.1088/1742-6596/1388/1/012013 Retrieved from www.scopus.com

Cagnon, B., Chatelain, S., de Oliveira, T. F., Versaveau, F., Delpeux, S., \& Chedeville, O. (2017). Adsorption of phthalates on activated carbons in monosolute solution and in mix within complex matrices. Water, Air, and Soil Pollution, 228(4) doi:10.1007/s11270-017-3315-7 
Cunha, G. D. S., Souza-Chaves, B. M. D., Bila, D. M., Bassin, J. P., Vecitis, C. D., \& Dezotti, M. (2019). Insights into estrogenic activity removal using carbon nanotube electrochemical filter. Science of the Total Environment, 678, 448456. doi:10.1016/j.scitotenv.2019.04.342

Cupertino, K. F., Prata, A. A., Le-Minh, N., Stuetz, R. M., \& Santos, J. M. (2020). Comparison of mass transfer parameters inside a USEPA flux hood for two VOCs. Water Science and Technology, 81(7), 1445-1451. doi:10.2166/wst.2020.197

D’Avila, J. V., Martinazzo, A. P., Dos Santos, F. S., Teodoro, C. E. S., \& Portz, A. (2016). Essential oil production of lemongrass (cymbopogon citratus) under organic compost containing sewage sludge. [Produção de óleo essencial de capim-limão (Cymbopogon citratus) sob composto orgânico contendo lodo de esgoto] Revista Brasileira De Engenharia Agrícola e Ambiental, 20(9), 811-816. doi:10.1590/1807-1929/agriambi.v20n9p811-816

Da Costa, J. F., Martins, W. L. P., Seidl, M., \& Von Sperling, M. (2015). Role of vegetation (typha latifolia) on nutrient removal in a horizontal subsurface-flow constructed wetland treating UASB reactor-trickling filter effluent. Water Science and Technology, 71(7), 1004-1010. doi:10.2166/wst.2015.055

Da Mata, R. A., Silva, C. M., Zanuncio, J. C., \& Materazzi, L. B. (2019). Effects of electrostatic precipitators ash leachate (EPAL) from recovery boilers on the biological treatment of effluent of kraft pulp mills. Science of the Total Environment, 659, 905-911. doi:10.1016/j.scitotenv.2018.12.413 